\title{
2D AND 3D DOPING OF SILICON MEMS STRUCTURES USING PHOSPHORUS- DOPED POLYSILICON AS A DOPANT SOURCE
}

Pascal J. Newby ${ }^{{ }^{*}}$, Kazem Zandi ${ }^{1}$, Karel Coté ${ }^{1}$, Jean-Philippe Richard ${ }^{1}$, and Khaled-Amir Belarbi ${ }^{2}$

${ }^{1}$ MiQro Innovation Collaborative Centre (C2MI), 45 boulevard de 1'Aéroport, J2L 1S8, Bromont, Qc, Canada

${ }^{2}$ Teledyne DALSA Semiconductor Inc., 18 boulevard de l'Aéroport, J2L 1S7, Bromont, Qc, Canada

\begin{abstract}
We use in-situ phosphorus-doped LPCVD (low-pressure chemical vapour deposition) polysilicon as a dopant source for silicon wafers. We used this technique for "3D doping", where poly-Si is deposited over structures patterned in silicon-on-insulator (SOI) wafers, which enables higher doping levels, as diffusion is performed through three surfaces rather than one. This technique can also be used for doping of unpatterned wafers, and we have derived a relation which estimates the doping level achieved with 3D doping using only 4-point probe measurements on blanket wafers. The advantage of this doping method compared to standard methods is that it does not require dedicated equipment and can be carried out using tools commonly available in a MEMS fab.
\end{abstract}

\section{INTRODUCTION}

Besides being used as a mechanical material in MEMS, silicon often also serves as an electrical conductor, so its resistivity must be tuned according to the application. There are many standard methods for ex-situ doping of silicon, such as ion implantation, $\mathrm{POCl}_{3}$, or spin-on glass, but these methods all require dedicated and expensive equipment which may not be available in a MEMS fab. As an alternative to these techniques, we propose to use in-situ phosphorus-doped polysilicon as a dopant source. Once deposited, the phosphorus can be transferred from the poly-Si to the silicon wafer by annealing, and the advantage of this technique is that it uses equipment readily available in a MEMS fab [1].

Polysilicon has been used before as a dopant source in the $80 \mathrm{~s}$ and early $90 \mathrm{~s}$ [2]-[4] for transistor fabrication. In this previous work poly-Si was not in-situ doped, but doped after deposition, generally by ion implantation [3], [4], and the focus was on using poly-Si for the fabrication of ultra-shallow junctions in MOSFETs or emitters in bipolar transistors [4]-[6]. Work on the use of poly-Si as a dopant source for MEMS applications has never been published.

We will present the development and optimization of the process, and apply this process to doping of both unpatterned and patterned wafers. The latter process will be referred to as "3D doping". We will also show how doping of unpatterned wafers can be used to predict doping of patterned wafers and discuss the issues encountered during the development of this process.

\section{D DOPING}

In this article we mainly focus on 3D doping of SOI (siliconon-insulator) wafers. Wafers are patterned and the device layer is etched down to the buried oxide layer, and poly-Si is deposited over the patterned structure. The wafers are then annealed, as illustrated in Figure 1, to transfer dopant atoms to the wafer. The advantage of this technique, compared to $2 \mathrm{D}$ doping, is that as doping is performed through three surfaces, much higher doping levels can be reached than with $2 \mathrm{D}$ doping for the same thermal budget, and this is especially true for high aspect ratio structures.

\section{EXPERIMENTAL DETAILS}

All wafers used were $200 \mathrm{~mm}$ in diameter with $\{100\}$ orientation. The bulk wafers were $725-\mu$ m-thick. The SOI wafers had $25-\mu \mathrm{m}$-thick device layers and $2-\mu \mathrm{m}$-thick buried oxide. Both

978-1-940470-03-0/HH2018/\$25@2018TRF

DOI 10.31438/trf.hh2018.34 a) etch device layer of SOI wafer

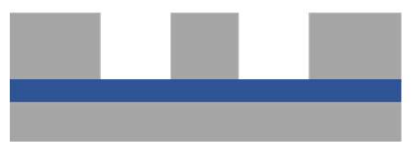

b) deposit poly-Si and diffuse dopants into wafer

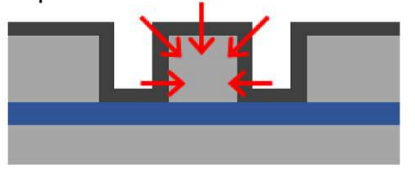

Figure 1: Basic principle of $3 D$ doping.

the bulk wafers and device layer of the SOI wafers had an initial resistivity of 1-10 $\Omega$.cm and were n-type phosphorus-doped.

The SOI wafers were etched by DRIE (deep reactive ion etching) down to the buried oxide layer. The test structures were double beams, each with a width of $7 \mu \mathrm{m}$ and lengths between 1 and $3 \mathrm{~mm}$, as shown in Fig. 2. Etched dimensions were measured using an optical microscope or SEM (scanning electron microscope).

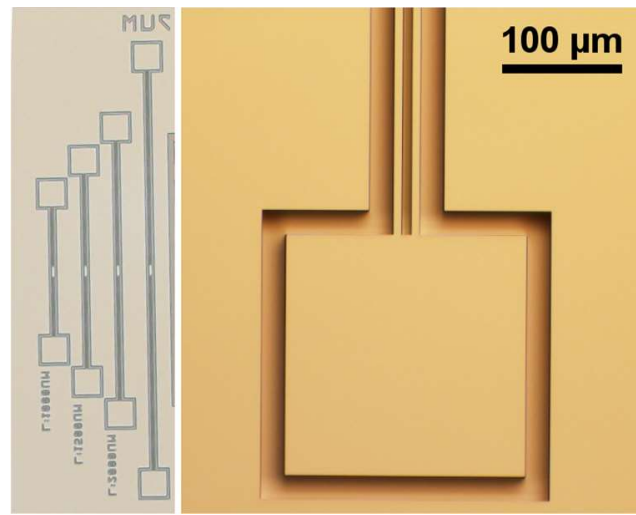

Figure 2: Optical microscope image showing the test structures etched in an SOI wafer by DRIE.

The polysilicon layers were deposited by low-pressure chemical vapour deposition (LPCVD). Doping of the poly-Si was carried out in-situ during the deposition so phosphine was used as well as silane. Oxidation of the poly-Si layers was performed by wet thermal oxidation at 1000 or $1100^{\circ} \mathrm{C}$. The oxidized poly-Si was etched in 10:1 hydrofluoric acid (HF).

Thickness of the oxide layers was measured by reflectometry using an n\&k Olympian. Thickness of the poly-Si layers was measured by reflectometry when they were above an oxide layer (over the buried oxide of a patterned SOI wafer for instance) and by weighing the mass change when they were deposited directly on silicon for the unpatterned wafers. Sheet resistance measurements were performed either with a Prometrix RS55 or with a 4-point probe mounted on a manual prober. Resistance measurements were done with a manual prober using a 4-wire configuration and a Keithley 2400 SMU. Average resistivity of the silicon in the structures is extracted from the resistance measurements.

Both within-wafer non-uniformity (WIWNU) and wafer-towafer non-uniformity (WTWNU) were calculated as (Max - Min)/ ( $2 *$ average) throughout this paper. Hilton Head Island, South Carolina, June 3-7, 2018 


\section{RESULTS AND DISCUSSION}

\section{Development of the Basic Process Flow}

Phosphorus-doped polysilicon is deposited over silicon wafers by LPCVD. Dopants must then be transferred from the poly-Si layer to the wafer, by annealing. It is also desirable to remove the poly$\mathrm{Si}$ after dopant transfer and before further processing, and selectivity between the single-crystal silicon substrate and poly-Si is an obvious challenge in this case. One option for removing poly-Si is a timed etch, either wet or plasma. Instead of this, we have chosen to thermally oxidize the poly-Si and then remove it using HF. This option was chosen as uniformity and repeatability are far better for thermal oxidation than for etching, meaning that poly-Si can be removed while minimizing the effect on the silicon substrate.

The poly-Si film can be oxidized before or after the dopant drive-in anneal, so we compared both options. Two SOI wafers with patterned device layers were used. After depositing the $1-\mu \mathrm{m}$-thick poly-Si layer, one wafer was oxidized before the drive-in anneal, and the other was oxidized after annealing. Table 1 summarizes the process steps. When annealing before oxidation, a thin cap-oxide must be created on the poly-Si before annealing to prevent dopant out-gassing [7]. The second oxidation $(0.4 \mu \mathrm{m})$ was performed as initial electrical measurements after the $2.2 \mu \mathrm{m}$ oxidation showed that there was still a thin layer of poly-Si left which caused a shortcircuit between the test structures and the rest of the wafer.

Table 1: Process steps used to determine optimal order for oxidation and drive-in anneal.

\begin{tabular}{|c|c|}
\hline $\begin{array}{l}\text { Oxidation after drive-in } \\
\text { anneal }\end{array}$ & $\begin{array}{l}\text { Oxidation before drive-in } \\
\text { anneal }\end{array}$ \\
\hline \multicolumn{2}{|c|}{ Photo + DRIE of SOI device layer } \\
\hline \multicolumn{2}{|c|}{ Deposit $1 \mu \mathrm{m}$ poly-Si } \\
\hline $\begin{array}{c}\text { Thermal cap oxide, } 15 \mathrm{~nm}+ \\
1 \mathrm{~h} \text { anneal } 1100^{\circ} \mathrm{C}\end{array}$ & $\begin{array}{l}\text { Thermal oxidation } 1100^{\circ} \mathrm{C}, \\
2.2 \mu \mathrm{m}\end{array}$ \\
\hline \multicolumn{2}{|c|}{ Drive-in anneal, $15 \mathrm{~h} 1100^{\circ} \mathrm{C}$} \\
\hline $\begin{array}{l}\text { Thermal oxidation } 1100^{\circ} \mathrm{C}, \\
2.2 \mu \mathrm{m}\end{array}$ & - \\
\hline \multicolumn{2}{|c|}{ Etch oxidized poly-Si in HF } \\
\hline \multicolumn{2}{|c|}{$2^{\text {nd }}$ thermal oxidation, $0.4 \mu \mathrm{m}$} \\
\hline \multicolumn{2}{|c|}{ Etch oxidized poly-Si in HF } \\
\hline
\end{tabular}

After final removal of the oxidized poly-Si, the resistance of the test structures was measured at 13 points across the wafer and the results are given in Table 2. The results show that oxidizing after the drive-in anneal leads to a marginally lower doping level, despite a slightly higher thermal budget. Furthermore, uniformity is better when oxidation is performed before annealing, and there is one less process step, so we chose to use this process.

The oxidation of poly-Si must be well controlled. Indeed, as mentioned above, after the first $2.2 \mu \mathrm{m}$ thermal oxidation, there was still some remaining unoxidized poly-Si. Conversely, oxidizing for too long will consume silicon from the substrate. Several oxidation runs of poly-Si were carried out with different oxidation times, and

Table 2: Resistance measurements on SOI wafers, where oxidation was performed either before or after the drive-in anneal.

\begin{tabular}{cccc}
\hline Process & $\begin{array}{c}\text { Average resistance } \\
{[\mathbf{m} \Omega . \mathbf{c m}]}\end{array}$ & $\begin{array}{c}\text { Range } \\
{[\mathbf{m} \Omega . \mathbf{c m}]}\end{array}$ & $\begin{array}{c}\text { WIWNU } \\
{[ \pm \%]}\end{array}$ \\
\hline $\begin{array}{c}\text { Oxidation } \\
\text { after anneal } \\
\begin{array}{c}\text { Oxidation } \\
\text { before anneal }\end{array}\end{array}$ & 1.19 & 0.14 & 6 \\
\hline
\end{tabular}

our measurements show that poly-Si is oxidized faster than singlecrystal $\langle 100\rangle$ silicon, but once oxidized behaves the same as oxidized single-crystal silicon and can be described using the DealGrove model [8]. To avoid issues due to incomplete oxidation of the poly-Si, the oxidation time was slightly extended so that about $90 \mathrm{~nm}$ of the silicon substrate beneath the poly-Si was consumed.

\section{Effect of Polysilicon Thickness}

Using the optimized process flow, with oxidation of the polySi before the drive-in anneal and the optimized oxidation time, both presented above, the doping process was repeated with a thinner poly-Si layer with a thickness of $0.34 \mu \mathrm{m}$ to compare with the results obtained for $1-\mu \mathrm{m}$ layers. Two SOI wafers were used, as well as several unpatterned test wafers. One SOI wafer was cleaved after the poly-Si deposition to observe the poly-Si film using SEM. Figure 3 shows that the film is conformal.

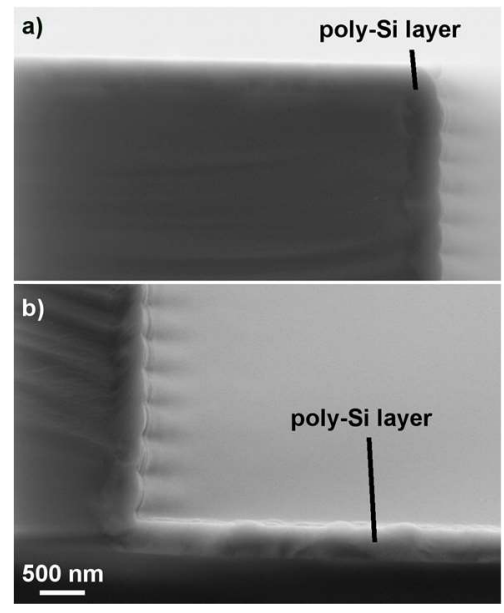

Figure 3: Cross-section SEM images of poly-Si layer deposited over a patterned SOI wafer.

The thickness of the as-deposited and oxidized poly-Si films was measured, as well as its resistivity (see experimental details). The resistance of the test structures on the SOI wafer was measured on 19 dies across the wafer. These results are summarized in Table 3, including data on within-wafer and wafer-to-wafer non-uniformity. In addition, two oxidation runs were performed, and there was a $1.2 \%$ difference between the average oxide thicknesses for both runs. For all the steps, wafer-to-wafer and within-wafer uniformity are excellent. The main source of within-wafer non-uniformity is the resistivity of the poly-Si, which is reflected in the WIWNU of the average resistivity of the patterned structures. Compared to the results with a $1-\mu \mathrm{m}$ poly-Si layer, the $0.34-\mu \mathrm{m}$ poly-Si layer contains a smaller amount of phosphorus, so as expected the resistivity obtained with the $0.34 \mu \mathrm{m}$ poly-Si layer is higher.

Table 3: Data for characterization of the main process steps.

\begin{tabular}{|c|c|c|c|}
\hline Measurement & Value & $\begin{array}{c}\text { WIWNU } \\
{[ \pm \%]}\end{array}$ & $\begin{array}{c}\text { WTWNU } \\
{[ \pm \%]}\end{array}$ \\
\hline Poly-Si thickness [nm] & 337 & 0.7 & 1.7 \\
\hline $\begin{array}{l}\text { Poly-Si resistivity } \\
{[\mathrm{mOhm} . \mathrm{cm}]}\end{array}$ & 1.82 & 4.8 & 0.7 \\
\hline Silicon oxide thickness & 952 & 0.3 & 0.1 \\
\hline $\begin{array}{l}\text { Average resistivity of } \\
\text { patterned structures }[\mathrm{m} \Omega . \mathrm{cm}]\end{array}$ & 3.9 & 4.0 & - \\
\hline
\end{tabular}




\section{Using 2D-Doped Wafers to Estimate 3D Doping Level}

In order to reduce development costs and time, we have derived a relation which uses only 4-point probe measurements of sheetresistance performed on 2D-doped unpatterned wafers to estimate the resistivity which would be obtained for 3D doping with the same thermal budget. This saves the photolithography and etch steps, and means the process can be developed using bulk wafers rather than costly SOI wafers.

\section{a) 2D doping of bulk wafer}

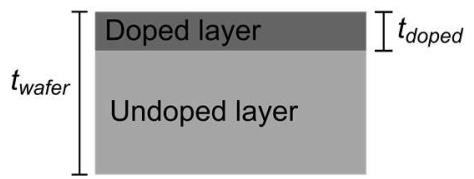

\section{b) 3D doping of patterned} SOI wafer

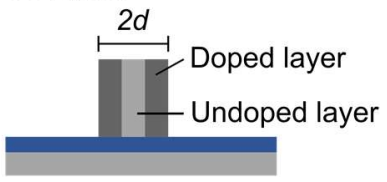

Figure 4: Cross-sections of geometries used to model a) 2D doping of a bulk wafer and b) $3 D$ doping of a patterned SOI wafer.

The first step is to estimate the sheet resistance of the doped layer from the measured sheet resistance of the whole wafer, without knowing the doping depth, as illustrated in Fig. 4a. The measured sheet resistance $R_{S \text {, meas 2D }}$ depends on the sheet resistance of the doped layer, $R_{S \text {, doped 2D }}$ and the undoped wafer, $R_{S \text {, undoped 2D }}$ :

$$
\frac{1}{R_{S, \text { meas 2D }}}=\frac{1}{R_{S, \text { doped 2D }}}+\frac{1}{R_{S, \text { undoped 2D }}}
$$

The sheet resistance of the undoped part of the wafer is $R_{s}=\left(t_{\text {wafer }}-t_{\text {doped }}\right) / \rho_{0}$, where $t_{\text {wafer }}$ is the thickness of the wafer and $t_{\text {doped }}$ the thickness of the doped layer, and $\rho_{0}$ is the initial resistivity of the wafer. The thickness of the doped layer is unknown but on the order of a few microns, so can be neglected compared to the total thickness of the wafer $(725 \mu \mathrm{m})$, which allows us to express the sheet resistance of the doped layer as a function of known values:

$$
\frac{1}{R_{S, \text { doped 2D }}} \approx \frac{1}{R_{S, \text { meas 2D }}}-\frac{t_{\text {wafer }}}{\rho_{0}}
$$

We will now use this value to estimate the average resistivity of a 3D doped structure. Our structures have a fairly high aspect ratio, so we will consider only lateral doping. In this case we can represent the doped structure as two doped layers, one on each side of a central undoped layer (see Figure 4b). The sheet resistance of each doped layer is equal to that of the 2D-doped layer calculated in (2), so $R_{S \text {, doped 3D }}=R_{S \text {, doped 2D }}$. The total sheet resistance of the doped beams, $R_{s, \text { beam }}$, is:

$$
\frac{1}{R_{s, \text { beam }}}=\frac{2 d}{\rho_{\text {avg 3D }}}=\frac{2}{R_{s, \text { doped 3D }}}+\frac{1}{R_{s, \text { undoped 3D }}}
$$

where $d$ is the half-width of the beam and $\rho_{\text {avg }}$ the average resistivity of the beam. The thickness of the doped and undoped layers are comparable and the resistivity of the doped layer is orders of magnitude smaller than that of the undoped layer, meaning that $1 / R_{s, \text { undoped }} \ll 1 / R_{s, \text { doped }}$. So using the expression for $R_{s \text {, doped }}$ calculated in (2) the average resistivity of the beam is:

$$
\rho_{\text {avg 3D }}=d * R_{s, \text { doped 3D }}=\frac{d}{\left(\frac{1}{R_{s, \text { meas 2D }}}-\frac{t_{\text {wafer }}}{\rho_{0}}\right)}
$$

We used (4) to estimate $3 \mathrm{D}$ resistivities using data from 2Ddoped wafers and compared these with resistivities measured on 3Ddoped wafers. 2D sheet resistance data for the $0.34 \mu \mathrm{m}$ poly-Si layer was obtained from two bulk unpatterned wafers which were processed at the same time as the SOI wafer. For the tests presented at the beginning of the results section, with $1 \mu \mathrm{m}$ of poly-Si, there were no unpatterned wafers, but 4-point probe measurements were taken on unpatterned areas of the SOI wafers. Table 4 shows sheet resistance values and average $3 \mathrm{D}$ resistivity estimated from these values. The last column repeats the measured $3 \mathrm{D}$ resistivities from previous sections and shows that the agreement between the estimated and measured 3D resistivities is very good. The data for the 1- $\mu \mathrm{m}$ poly-Si was taken from unpatterned areas of SOI wafers with $25-\mu \mathrm{m}$-thick device layers, which cannot be considered as infinitely thick compared to the doped layer. This may be a reason why the agreement is not quite as good for $1-\mu \mathrm{m}$ poly-Si.

\section{Issues}

Some minor issues were observed during the development of this process. The first issue is an increase in the roughness of the silicon surface, which appears during the oxidation of the poly-Si layer and remains after removal of the oxidized poly-Si. The roughness was not measured, but is clearly observable under the microscope, as shown in Fig. 5. A possible explanation for this is phosphorus precipitation at the $\mathrm{Si} / \mathrm{SiO}_{2}$ interface during oxidation.

The doping process also led to the formation of dislocations. In Figure 5 we can see lines following the etched edges, which follow the $<110\rangle$ crystal direction. The lines are approximately $18 \mu \mathrm{m}$ away from the etched edges, which is consistent with a dislocation propagating from the bottom corner of an etched beams along a $\{111\}$ plane. Like the roughness, the dislocations appeared during the oxidation of the poly-Si and were observed for both the

\begin{tabular}{|c|c|c|c|c|c|}
\hline Wafer & $\begin{array}{c}\text { Measured 2D sheet } \\
\text { resistance } \boldsymbol{R}_{\boldsymbol{s}} \text {, meas 2D } \\
{[\mathrm{Ohm} / \mathrm{sq}]}\end{array}$ & $\begin{array}{c}\text { WIWNU } \\
R_{s, \text { meas 2D }} \\
\quad[ \pm \%]\end{array}$ & $\begin{array}{c}\text { Calculated } \\
\boldsymbol{R}_{\boldsymbol{s}, \text { doped 2D }} \\
{[\mathbf{O h m} / \mathbf{s q ]}}\end{array}$ & $\begin{array}{l}\text { Estimated average } \\
\text { 3D resistivity } \\
\text { [mOhm.cm] }\end{array}$ & $\begin{array}{l}\text { Measured average } \\
\text { 3D resisitivity } \\
\text { [mOhm.cm] }\end{array}$ \\
\hline Oxidation after anneal, $1 \mu \mathrm{m}$ poly-Si & 4.69 & 3.4 & 4.71 & 1.41 & 1.19 \\
\hline Oxidation before anneal, $1 \mu \mathrm{m}$ poly-Si & 4.63 & 3.2 & 4.64 & 1.39 & 1.14 \\
\hline $0.34 \mu \mathrm{m}$ poly-Si wafer 1 & 9.37 & 1.5 & 12.1 & 3.93 & \multirow{2}{*}{3.87} \\
\hline $0.34 \mu \mathrm{m}$ poly-Si wafer 2 & 9.52 & 1.7 & 12.4 & 4.02 & \\
\hline
\end{tabular}
0.33 and $1-\mu \mathrm{m}$ poly-Si layers. The most likely explanation is the

Table 4: Sheet resistance measured on unpatterned doped wafers and 3D average resistivity estimated from these values. The last column contains measured $3 D$ resistivities copied from the previous section for ease of comparison. 


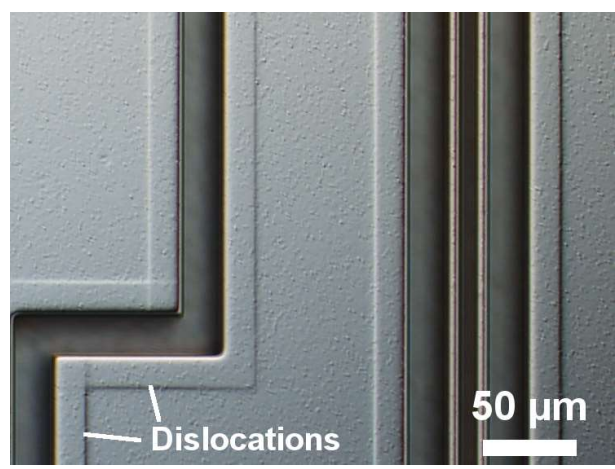

Figure 5: DIC (differential interference contrast) microscope image of a wafer after removing the oxidized poly-Si. The surface roughness and dislocations caused by the doping process are visible.

compressive stress created in the poly-Si layer when it is oxidized. The height of the dislocation was measured by SEM and white-light interferometry and the measured values were between 50 and $90 \mathrm{~nm}$.

The final issue is the presence of a residue of unoxidized poly$\mathrm{Si}$ at the foot of the beams. Fig. 6 shows a cross-section SEM image of a beam after etching of the oxidized poly-Si, and the unoxidized poly-Si is clearly visible at the foot of the beam. It has been shown in the literature that oxidation of silicon is slower around corners due to compressive stress [9]. Whether the three issues presented are a problem or not will depend on each application.

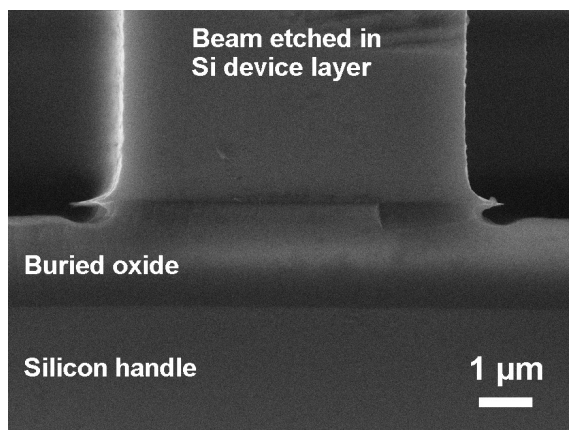

Figure 6: Cross-section SEM image of a beam patterned in the device layer of an SOI wafer after removal of the oxidized poly-Si.

\section{Advantages of Polysilicon Doping}

Using polysilicon as a dopant source has several advantages compared to other doping techniques. Standard ex-situ doping techniques such as ion implantation, $\mathrm{POCl}_{3}$, and spin-on dopant all require dedicated equipment which may not be available in a typical MEMS fab. Polysilicon doping on the other hand, requires only a poly-Si furnace and an oxidation furnace, which are common tools in a MEMS fab.

Another solution for obtaining doped SOI wafers is simply to purchase wafers with the desired initial doping. However typical within-wafer variation is around $\pm 5 \%$ and wafer-to-wafer variation is generally around $\pm 10 \%$, unless the wafers are binned into very small lots, which will increase cost. We do not have wafer-to-wafer data for the final resistivity for poly-Si doping, but it should be commensurate with the wafer-to-wafer variations for the previous steps presented in Table 3, so should be able to achieve better nonuniformities than that of doped SOI wafers.

Furthermore, poly-Si doping enables 3D doping, which can be used to reach lower resistivities for the doped structures, given that three surfaces rather than one are used for introducing dopants. Indeed, 3D doping is not possible with ion implantation, and might be feasible with spin-on dopants, but only for limited step heights.

\section{CONCLUSION}

We have developed a technique for doping of silicon wafers using in-situ phosphorus-doped LPCVD polysilicon. The poly-Si can be deposited over either unpatterned or patterned wafers, and in the latter case, has the advantage of being able to reach higher doping levels due to the higher surface involved. The poly-Si is thermally oxidized to allow selective removal in HF from the silicon wafers, and we have shown that the process yields better results when oxidation is performed before the drive-in anneal rather than after. Finally, we have established a relation between the sheet resistance of 2D doped wafers and the resistivity of patterned wafers, which can be used to reduce development time and costs.

The main advantage of this technique compared to other doping methods is that it can be performed with equipment available in a typical MEMS fab. Within-wafer and wafer-to-wafer uniformity are excellent, and providing that run-to-run uniformity is as good, this method should be usable in industrial fabrication process-flows.

Future work includes performing more runs to gather data on run-to-run repeatability and varying the thermal budget more to study the effect on final resistivity, as well as resolving the issues with the process which were observed.

\section{ACKNOWLEDGEMENTS}

The authors would like to thank the staff of Teledyne Dalsa for their help in the fabrication of the wafers used in this work.

\section{REFERENCES}

[1] M. Biebl, G.T. Mulhern, and R.T. Howe, "In Situ Phosphorusdoped Polysilicon For Integrated MEMS," Proceedings of the International Solid-State Sensors and Actuators Conference TRANSDUCERS '95, Stockholm, Sweden, 6/25-29/95, IEEE (1995), pp, 198-201.

[2] W.J.M.J. Josquin, P.R. Boudewijn, and Y. Tamminga, "Effectiveness of polycrystalline silicon diffusion sources," Appl. Phys. Lett., 43, 960 (1983).

[3] H. Schaber, R. v. Criegern, and I. Weitzel, "Analysis of polycrystalline silicon diffusion sources by secondary ion mass spectrometry,” J. Appl. Phys., 58, 4036 (1985).

[4] K. Park, S. Batra, S. Banerjee, G. Lux, and T.C. Smith, "Analysis of ion-implanted amorphous and polycrystalline silicon films as diffusion sources for ultrashallow junctions," J. Appl. Phys., 70, 1397 (1991).

[5] T.I. Kamins, "Effect of polysilicon-emitter shape on dopant diffusion in polysilicon-emitter transistors," IEEE Electron Device Lett., 10, 401 (1989).

[6] G.E. Georgiou, T.T. Sheng, F.A. Baiocchi, J. Kovalchick, W.T. Lynch, and D. Malm, "Shallow junctions by outdiffusion from Arsenic implanted polycrystalline silicon," J. Appl. Phys., 68, 3714 (1990).

[7] S.P. Murarka, "Phosphorus out-diffusion during high temperature anneal of phosphorus-doped polycrystalline silicon and SiO2," J. Appl. Phys., 56, 2225 (1984).

[8] B.E. Deal and A.S. Grove, "General relationship for the thermal oxidation of silicon," J. Appl. Phys., 36, 3770 (1965).

[9] R.B. Marcus and T.T. Sheng, "The Oxidation of Shaped Silicon Surfaces,” J. Electrochem. Soc., 129, 1278 (1982).

\section{CONTACT}

*P.J. Newby, Pascal.Newby@,c2mi.ca 